\title{
Association of In-Hospital Surgical Bleeding Events with Prolonged Hospital Length of Stay, Days Spent in Critical Care, Complications, and Mortality: A Retrospective Cohort Study Among Patients Undergoing Neoplasm-Directed Surgeries in English Hospitals
}

This article was published in the following Dove Press journal: ClinicoEconomics and Outcomes Research

\begin{abstract}
Stephen S Johnston'
Nadine Jamous ${ }^{2}$

Sameer Mistry ${ }^{3}$

Simran Jain ${ }^{4}$

Gaurav Gangoli ${ }^{5}$

Walter Danker ${ }^{5}$

Eric Ammann ${ }^{6}$

Kingsley Hampton ${ }^{7}$

'Department of Epidemiology, Medical Devices, Johnson \& Johnson, New Brunswick, NJ, USA; ${ }^{2}$ Department of Health Economics and Market Access, Johnson \& Johnson Medical Ltd, Edinburgh, UK; ${ }^{3}$ Department of Medical Affairs, Johnson \& Johnson Medical Ltd, Berkshire, UK; ${ }^{4}$ Department of Decision Science, Mu Sigma, Bangalore, India; ${ }^{5}$ Department of Health Economics and Market Access, Ethicon, Inc, Somerville, NJ, USA; ${ }^{6}$ Department of Epidemiology, Janssen, Titusville, NJ, USA; ${ }^{7}$ Department of Cardiovascular Science, University of Sheffield, Sheffield, UK
\end{abstract}

Correspondence: Stephen S Johnston Department of Epidemiology, Medical Devices, Johnson \& Johnson, 410 George Street, New Brunswick, NJ 0890I, USA Tel +l-443-254-2222

Email sjohn147@its.jnj.com
Purpose: To evaluate the association of in-hospital surgical bleeding events with the outcomes of hospital length of stay (LOS), days spent in critical care, complications, and mortality among patients undergoing neoplasm-directed surgeries in English hospitals.

Patients and Methods: This is a retrospective cohort study using English hospital discharge data (Hospital Episode Statistics [HES]) linked to electronic health records (Clinical Practice Research Datalink [CPRD]). HES includes information on patient demographics, admission and discharge dates, diagnoses and procedures, days spent in critical care, and discharge status. CPRD includes information on patient demographics, diagnoses and symptoms, drug exposures, vaccination history, and laboratory tests. Patients aged $\geq 18$ years who underwent selected neoplasm-directed surgeries between 1-Jan-2010 and 29-February-2016: hysterectomy, low anterior resection (LAR), lung resection, mastectomy, and prostate surgery were included. The primary independent variable was in-hospital surgical bleeding events identified by diagnosis of haemorrhage and haematoma complicating a procedure or reopening/re-exploration and surgical arrest of postoperative bleeding. Outcomes included LOS, days spent in critical care, in-hospital complications (diagnoses of infections, acute renal failure, vascular events), and in-hospital mortality, identified during surgery through discharge. Multivariable regression was used to examine the adjusted association of bleeding events with outcomes.

Results: The study included 26,437 neoplasm-directed surgeries (hysterectomy=6092; $\mathrm{LAR}=2957$; lung=1538; mastectomy $=12,806$; prostate $=3044$ ). Incidence proportions of bleeding events were: hysterectomy $=1.9 \% \quad(95 \%$ confidence interval $=1.1-2.5 \%)$; $\mathrm{LAR}=3.0 \%(\mathrm{CI}=2.3-3.6 \%)$; lung $=1.8 \%(\mathrm{CI}=1.1-2.5 \%)$; mastectomy $=1.6 \%(\mathrm{CI}=1.3-1.8 \%)$; prostate $=1.0 \%(\mathrm{CI}=0.6-1.3 \%)$. In adjusted analyses, bleeding events were associated with: prolonged LOS: $3.1(\mathrm{CI}=1.1-6.3)$ mastectomy to $5.7(\mathrm{CI}=3.6-8.2)$ LAR days longer; more days spent in critical care: $0.4(\mathrm{CI}=0.03-0.27)$ mastectomy to $6.5(\mathrm{CI}=2.5-13.6)$ hysterectomy days more; and higher incidence proportions of all examined complications; all $P<0.05$. Conclusion: This study quantifies a substantial clinical and healthcare resource utilization burden associated with surgical bleeding among patients undergoing neoplasm-directed surgery in England hospitals. 
Keywords: hysterectomy, low anterior resection, lung resection, mastectomy, prostate surgery, hospital episode statistics admitted patient care database, clinical practice research datalink GOLD database

\section{Introduction}

Surgical bleeding events are a significant cause of morbidity, mortality, and economic burden on healthcare systems. ${ }^{1-3}$ Patients who are undergoing neoplasm-directed surgery may be prone to surgical bleeding due to a wide variety of factors, including neoadjuvant therapies, use of anticoagulants, tumor vascularity, and the complexity and anatomical location of surgical resection, among others. ${ }^{4-6}$

There is presently a paucity of literature regarding the incidence of surgical bleeding events and their association with clinical and economic outcomes among patients in England. Such information may be useful to inform National Health Service (NHS) and hospital-level decisions regarding the potential benefits of investment in improving the prevention and mitigation of surgical bleeding.

Therefore, we conducted a population-based study to evaluate the association of in-hospital surgical bleeding events with the outcomes of post-surgical hospital length of stay and post-surgical days spent in critical care among patients undergoing the following common neoplasmdirected surgeries, representing multiple specialties, in English hospitals: low anterior resection, hysterectomy, lung resection, mastectomy, and prostate (prostatectomy and resection). We hypothesized that patients with such bleeding events would experience prolonged post-surgical hospital length of stay and more post-surgical days spent in critical care than those without such events.

To examine potential underlying clinical mechanisms by which bleeding events may be associated with length of stay and days spent in critical care, we also evaluated the association of bleeding events with (a) in-hospital complications related to bleeding or transfusion, and (b) inhospital mortality. Complications of interest included infections, acute renal failure, and vascular events. ${ }^{7-11}$

\section{Methods}

\section{Data Sources}

We conducted this retrospective study using de-identified health records from two linked databases: the Hospital Episode Statistics Admitted Patient Care (HES-APC) database and the Clinical Practice Research Datalink (CPRD) GOLD database. ${ }^{12,13}$ The linkage of these two databases allowed us to measure the in-hospital outcomes of patients (from HES-APC) while accounting for a broad set of patient factors, such as resource use and medications taken, measured prior to admission to the hospital (from CPRD GOLD).

The HES-APC data are collected from all admissions/ attendances at NHS hospitals in England, including acute hospital trusts, primary care trusts, and mental health trusts. The HES-APC database includes information on patient demographics, admission and discharge dates, diagnoses recorded (including the primary diagnosis), procedures performed, days spent in critical care, and discharge status. ${ }^{14}$ Diagnoses are recorded using International Classification of Diseases version 10 (ICD10) codes. Procedures are recorded using the United Kingdom (UK) Office of Population, Census and Surveys classification (OPCS) 4.6 codes.

Broadly, CPRD data are collected from a network of over 1500 primary care practices in the UK; the CPRD GOLD data are collected from general practices specifically using the Vision electronic health record software. The CPRD GOLD database includes information on patient demographics, diagnoses and symptoms, drug exposures, vaccination history, laboratory tests, and referrals to hospital and specialist care. ${ }^{15}$ Diagnoses are recorded using Read codes and medcodes. Drugs are recorded using Gemscript codes.

In the most recent (June 2018) linkage, 10.6 million patients from 411 general practices in England were present from the CPRD GOLD database, of whom 8,444,946 were eligible for linkage to the HES-APC database and $7,754,885$ were ultimately linked. ${ }^{13}$ The CPRD GOLD data and linkages have been widely used for epidemiologic and healthcare research and form the basis of over 2200 peer-reviewed publications. ${ }^{16} \mathrm{~A}$ complete listing of codes used for this study is provided in the Appendix.

\section{Research Approval and Protection of Human Subjects}

We obtained the de-identified HES-APC and CPRD study data under license from the UK Medicines and Healthcare Products Regulatory Agency (MHRA). Throughout the study, we stored the data on encrypted, password-protected servers. The Independent Scientific Advisory Committee for 
MHRA Database Research reviewed and approved the study protocol (number 17_091R). The interpretation and conclusions contained in this report are those of the authors alone.

\section{Patient and Public Involvement}

Patients or the public were not involved in the design, or conduct, or reporting, or dissemination plans of our research.

\section{Patient Selection Criteria}

We extracted data from the HES-APC for patients who underwent low anterior resection, hysterectomy, lung resection, mastectomy, or prostate surgery (prostatectomy and resection) between January 1, 2010, and February 29, 2016. For each patient, we identified the first admission in which a surgery of interest took place, designating it as the "index admission," and required that a diagnosis code related to neoplasm was recorded in the primary diagnosis position. We further required that patients were aged $\geq 18$ years as of the index admission and were registered with a general practitioner (GP) whose office contributed data to the CPRD GOLD for the 12 months prior to the index admission.

\section{Measurement of Bleeding Events and Study Outcomes}

In the HES-APC, hospital admissions are sub-divided into sequential periods of care - known as "episodes" - each carrying its own respective diagnosis and procedure codes. We classified patients as having experienced a bleeding event if, for the surgical episode or a subsequent episode within the index admission, either of the following was documented: (a) a diagnosis code for haemorrhage and haematoma complicating a procedure (ICD-10 T81.0); or (b) a procedure code for reoperation for surgical arrest of postoperative bleeding (OPCS T032, T301, Y321). We were unable to identify blood product transfusions in this study because blood product transfusions are not well captured in the HES-APC database. ${ }^{17}$

We defined post-surgical length of stay (LOS) as the time interval in days from the surgery of interest to the day of discharge from the index admission. We also calculated the number of such LOS days spent in critical care.

We searched for documented diagnosis codes for complications related to bleeding or transfusion (infection, acute renal failure, and vascular events) during the same time period in which we identified bleeding events; although we did not strictly require the diagnosis of bleeding events to occur before the diagnosis of complications among patients with bleeding events, $95.8 \%$ of bleeding events were identified during the surgical episode and it is possible that a complication may develop prior to the need for surgical arrest of post-operative bleeding. Infections of interest included cellulitis, pneumonia, sepsis, urinary tract infection, wound infection. Acute renal failure had no subcomponents. Vascular events included cerebrovascular accident, myocardial infarction, venous thromboembolism, and sudden death due to cardiac arrest.

Finally, we defined in-hospital mortality as a discharge status of "Died" (vs another discharge status) for the index admission; other discharge statuses included either "Discharged on clinical advice or with clinical consent" (accounting for 99.8\% of the remaining discharges) or "Self discharged, or discharged by a relative or advocate" (accounting for $0.2 \%$ of the remaining discharges).

\section{Measurement of Covariates}

We used HES-APC and CPRD GOLD records from the index admission and 12 months prior to measure covariates for the purpose of characterizing the study cohorts and estimating the adjusted association of bleeding events with the study outcomes, as described further below. Covariates included the following categories: patient demographics (age, sex, geographic region [East of England, London, Midlands, North, South Central, South East Coast, or South West]); index admission surgery type (low anterior resection, hysterectomy, lung surgery, mastectomy, or prostate surgery); index admission primary ICD-10 diagnosis type (malignant neoplasm of the primary site, other neoplasm, or benign neoplasms, carcinoma in situ, and neoplasms of uncertain behavior); surgical approach (open or minimally invasive); index admission admitting status (elective, non-elective/emergency, or other); comorbidities $^{18}$ (anemia, cerebrovascular disease, chronic pulmonary disease, chronic renal disease, congestive heart failure, connective tissue or rheumatic disease, dementia, diabetes, liver disease, myocardial infarction, peptic ulcer disease, peripheral vascular disease); drugs with known effects on bleeding risk (anticoagulants, antiplatelets, selective serotonin reuptake inhibitors [SSRIs], selective norepinephrine reuptake inhibitors [SNRIs]); measures of utilization and overall health status (count of distinct prescription medications taken, count of face-to-face primary care consultations, and prior hospitalization). The comorbidities and measures of utilization and overall health 
status were extracted from the CPRD GOLD and HESAPC data for the 12 months prior to the index admission.

\section{Statistical Analyses}

For each surgery group, we calculated the crude (unadjusted) incidence proportions of bleeding events, complications, and mortality as the number of patients with a given event divided by the number of patients in the surgery group. No formal statistically adjusted comparisons were made between the surgery groups, and therefore differences in the incidence proportions of bleeding events across surgeries reflect underlying differences in patient characteristics of the surgery groups. We used standardized differences (StdDiff) to compare the baseline characteristics of patients with vs without bleeding events; StdDiff are a sample-size-agnostic measure of substantive imbalance between groups and therefore are not prone to type 1 (type 2) error in the presence of large (small) sample sizes. ${ }^{19}$ When comparing unadjusted outcomes between groups, we used the Wilcoxon rank-sum (Mann-Whitney) test for LOS and days spent in critical care, and chi-squared tests for complications and mortality events. When examining the multivariable-adjusted association of bleeding events with LOS and days spent in critical care, we conducted a separate statistical analysis for each of the five surgery groups. However, complications and mortality events were infrequent, which necessitated that we pool all patients from across the surgery groups when analyzing these outcomes. We used multivariable generalized linear models with link functions and error distributions chosen based on statistical procedures to determine the appropriate selections. ${ }^{20,21}$ All models used the same a priori specification, which comprised all covariates described in the section above; no variable selection or reduction techniques were applied. The pooled models for complications and mortality included a categorical variable for surgery group. We used marginal standardization (recycled prediction) to generate multivariable adjusted estimates of LOS, days spent in critical care, and incidence proportions of complications and mortality for patients with vs without bleeding events, as well as the mean incremental difference (MID) in outcomes between those groups and the $95 \%$ confidence interval thereof. ${ }^{22}$ We performed the statistical analyses with SAS Enterprise Guide 7.1 for Windows (SAS Institute, Inc.; Cary, NC) and set $P<0.05$ as the threshold for statistical significance.

\section{Sensitivity Analyses}

After descriptive examination of the baseline factors by which patients with versus without bleeding events differed, we conducted a post-hoc sensitivity analysis wherein we repeated separate analyses of the study outcomes in patients with vs without baseline use of antiplatelet medications.

\section{Results}

The study included 26,437 eligible patients: 6092 hysterectomy, 2957 low anterior resection, 1538 lung surgery, 12,806 mastectomy, and 3044 prostate surgery. Appendix Supplementary Table 1 shows the reductions in study cohort size associated with the application of each patient selection criterion.

\section{Incidence of Bleeding Events}

Figure 1 shows the crude incidence proportion of bleeding events during the index admission for each surgery group. The crude incidence proportions of bleeding events were: $3.0 \%(95 \%$ confidence interval $[\mathrm{CI}]=2.3-3.6 \%)$ for low anterior resection, $1.9 \%(95 \% \mathrm{CI}=1.6-2.3 \%)$ for hysterectomy, $1.8 \%$, (95\% CI $=1.1-2.5 \%)$ for lung surgery, $1.6 \%(95 \% \mathrm{CI}=1.3-1.8 \%)$ for mastectomy, and $1.0 \%$ $(95 \% \mathrm{CI}=0.6-1.3 \%)$ for prostate surgery.

With respect to bleeding event identification, reoperation for surgical arrest of bleeding was documented in $44.4 \%$ of lung surgery patients with a bleeding event, $21.9 \%$ hysterectomy, $5.1 \%$ mastectomy, $3.5 \%$ prostate surgery, and $0.0 \%$ low anterior resection. Overall, $98.2 \%$ of reoperations for surgical arrest of bleeding were preceded or accompanied by a diagnosis of haemorrhage and haematoma complicating a procedure; $95.8 \%$ of bleeding events were first documented during the surgical episode, $4.2 \%$ were documented during an episode thereafter during the index admission (see footnote in Figure 1 for surgery group-specific information).

\section{Patient Characteristics}

Tables 1 and 2 show patient characteristics and index admission and surgery characteristics, respectively. Patients with bleeding events during the index admission had higher baseline prevalence of comorbidities, selected medications (eg, antiplatelet use, $20.9 \%$ vs $13.3 \%$, StdDiff=20.3), and face-to-face primary care consultations (11 [9.7] vs 9.4 [8], StdDiff=14.6) as compared with patients without bleeding events. As gauged by the StdDiff, patients with bleeding events were relatively similar to those without bleeding events in terms of index 


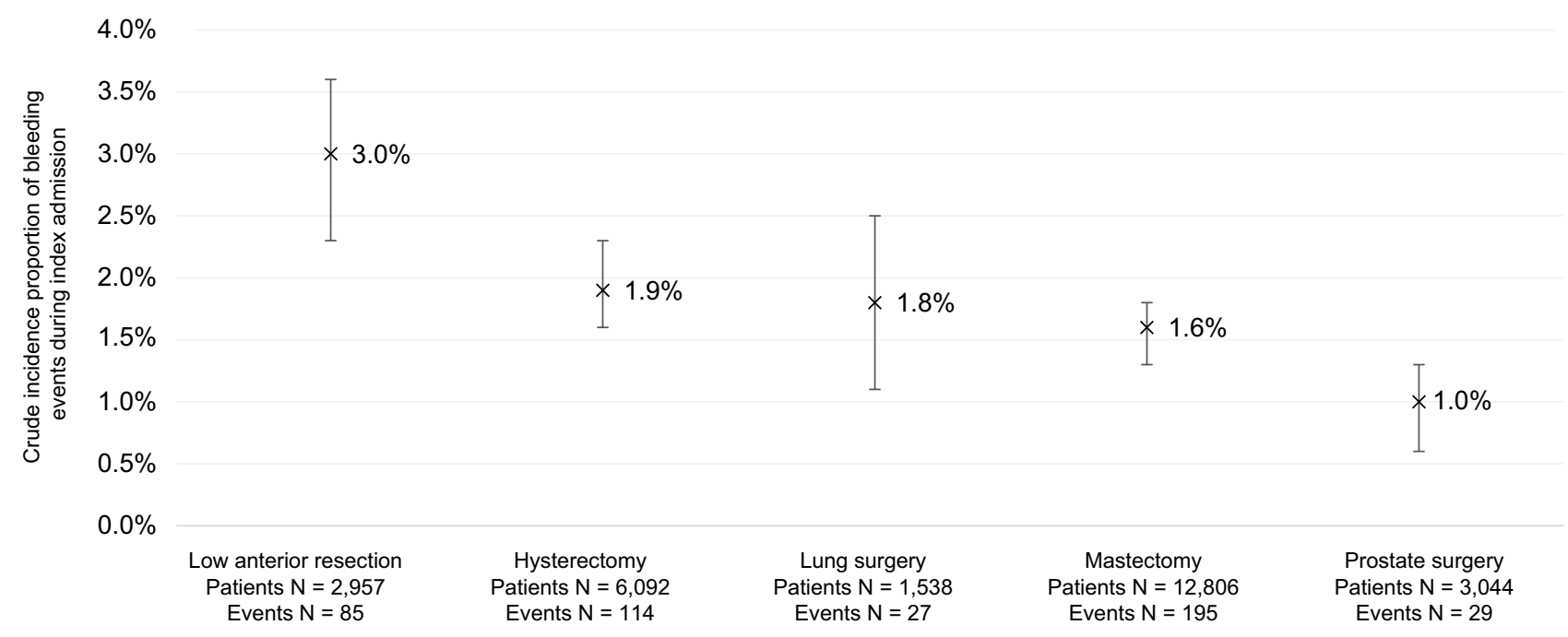

Figure I Crude incidence proportion of bleeding events during index admission*.

Notes: *The proportion of bleeding events first documented during the surgical episode was: $96.5 \%$ low anterior resection, $93.9 \%$ hysterectomy, $81.5 \%$ lung surgery, $97.9 \%$ mastectomy, and $100.0 \%$ prostate surgery; the proportion of patients with bleeding events who also had reoperation for surgical arrest of bleeding was: $0.0 \%$ low anterior resection, $21.9 \%$ hysterectomy, $44.4 \%$ lung surgery, $5.1 \%$ mastectomy, $3.5 \%$ prostate; only $1.8 \%$ of patients had reoperation for surgical arrest of bleeding without an accompanying diagnosis of haemorrhage and haematoma complicating a procedure ( 4 patients in the hysterectomy group, 4 patients in the lung surgery group); error bars represent $95 \%$ confidence intervals.

admission and surgery characteristics, aside from the differences in the incidence proportions of bleeding events across surgery groups described above.

\section{Association of Bleeding Events with LOS and Days Spent in Critical Care}

Figures 2 and 3 show the multivariable-adjusted mean LOS and days spent in critical care, respectively, for patients with vs without bleeding events in each surgery group; Appendix Supplementary Table 2 shows the corresponding unadjusted data. In all surgery groups, multivariable-adjusted mean LOS was statistically significantly longer for patients with bleeding events as compared with those without bleeding events (all $P<0.05)$ : MIDs ranged from 3.1 days $(95 \% \mathrm{CI}=1.1-6.3)$ for mastectomy to 5.7 days $(95 \% \mathrm{CI}=3.6-8.2)$ for low anterior resection. In congruence with LOS, the multivariable-adjusted mean number of days spent in critical care was statistically significantly longer for patients with bleeding events as compared with those without bleeding events (all $P<0.05$ ): MIDs ranged from 0.4 days $(95 \% \mathrm{CI}=0.03-2.7)$ for mastectomy to 6.5 days $(95 \% \mathrm{CI}=2.5-13.6)$ for hysterectomy.

\section{Association of Bleeding Events with In-Hospital Complications and In-Hospital Mortality}

Figure 4 shows multivariable-adjusted incidence proportions of in-hospital complications and in-hospital mortality for patients with vs without bleeding events in each surgery group; Appendix Supplementary Table 3 shows the corresponding unadjusted data. In all surgery groups, the multivariable-adjusted incidence proportion of in-hospital complications and in-hospital mortality was statistically significantly higher for patients with bleeding events as compared with those without bleeding events (all $P<0.05$ ): MIDs for the absolute percentage of patients with an event were $1.9 \%(95 \% \mathrm{CI}=0.7-4.0 \% ; 2.7 \%$ vs $0.8 \%)$ for acute renal failure, $2.2 \%(95 \% \mathrm{CI}=1.1-4.3 \% ; 2.8 \%$ vs $0.6 \%)$ for vascular events, $7.5 \%(95 \% \mathrm{CI}=4.5-11.6 \% ; 10.9 \% \mathrm{vs}$ $3.4 \%)$ for infections, and $1.9 \%(95 \% \mathrm{CI}=0.8-4.1 \% ; 2.3 \%$ vs $0.4 \%$ ) for in-hospital mortality.

\section{Sensitivity Analyses}

Results of the post-hoc sensitivity analyses of the study outcomes in patients with vs without baseline use of antiplatelet medications are reported in Appendix Supplementary Table 4. Overall, the crude incidence proportions of in-hospital bleeding events were numerically higher among patients with baseline antiplatelet use as compared with those without baseline antiplatelet use. With respect to LOS and critical care utilization, the mean incremental difference in outcomes between patients with vs without in-hospital bleeding events was generally larger in patients with baseline antiplatelet use as compared with those without baseline antiplatelet use; with the exception of findings among some of the smaller sub- 
Table I Patient Characteristics*

\begin{tabular}{|c|c|c|c|c|c|}
\hline Patient demographics & \multicolumn{2}{|c|}{$\begin{array}{l}\text { Patients without Bleeding Event } \\
N=25,987\end{array}$} & \multicolumn{2}{|c|}{$\begin{array}{l}\text { Patients with Bleeding } \\
\text { Event } N=450\end{array}$} & \multirow[t]{2}{*}{ StdDiff } \\
\hline \multirow{2}{*}{\multicolumn{6}{|c|}{ Age category, N/\% }} \\
\hline & & & & & \\
\hline $18-24$ & 333 & $1.3 \%$ & 2 & $0.4 \%$ & 9.8 \\
\hline $25-45$ & 3267 & $12.6 \%$ & 45 & $10.0 \%$ & 8.2 \\
\hline $46-64$ & 11,068 & $42.6 \%$ & 191 & $42.4 \%$ & 0.4 \\
\hline 65 Plus & 11,319 & $43.5 \%$ & 212 & $47.1 \%$ & -7.0 \\
\hline Female, N/\% & 20,467 & $78.7 \%$ & 352 & $78.2 \%$ & 1.5 \\
\hline \multicolumn{6}{|l|}{ Geographic region, N/\% } \\
\hline East of England & 2436 & $9.4 \%$ & 60 & $13.3 \%$ & -12.3 \\
\hline London & 3368 & $13.0 \%$ & 61 & $13.6 \%$ & -1.8 \\
\hline Midlands & 3627 & $14.0 \%$ & 51 & $11.3 \%$ & 8.1 \\
\hline North & 4521 & $17.4 \%$ & 79 & $16.0 \%$ & -1.6 \\
\hline South Central & 3837 & $14.8 \%$ & 66 & $14.7 \%$ & 0.3 \\
\hline South East Coast & 3985 & $15.3 \%$ & 58 & $12.9 \%$ & 6.9 \\
\hline South West & 3486 & $13.4 \%$ & 58 & $12.9 \%$ & 1.5 \\
\hline Yorkshire \& The Humber & 727 & $2.8 \%$ & 17 & $3.8 \%$ & -5.6 \\
\hline \multicolumn{6}{|l|}{ Comorbidities, N/\% } \\
\hline Anemia & 747 & $2.9 \%$ & 17 & $3.8 \%$ & -5.0 \\
\hline Cerebrovascular disease & 306 & $1.2 \%$ & 11 & $2.4 \%$ & -9.0 \\
\hline Chronic pulmonary disease & 4031 & $15.5 \%$ & 88 & $19.6 \%$ & -10.8 \\
\hline Chronic renal disease & 888 & $3.4 \%$ & 25 & $5.6 \%$ & -10.6 \\
\hline Congestive heart failure & 299 & $1.2 \%$ & 9 & $2.0 \%$ & -6.4 \\
\hline Connective tissue or rheumatic disease & 528 & $2.0 \%$ & 11 & $2.4 \%$ & -2.7 \\
\hline Dementia & 138 & $0.5 \%$ & 2 & $0.4 \%$ & 1.5 \\
\hline Diabetes & $387 !$ & $14.9 \%$ & 73 & $16.2 \%$ & -3.6 \\
\hline Liver disease & 290 & $1.1 \%$ & 13 & $2.9 \%$ & -12.9 \\
\hline Myocardial infarction & 222 & $0.9 \%$ & 6 & $1.3 \%$ & -3.8 \\
\hline Peptic ulcer disease & 120 & $0.5 \%$ & 3 & $0.7 \%$ & -2.6 \\
\hline Peripheral vascular disease & 393 & $1.5 \%$ & 15 & $3.3 \%$ & -11.8 \\
\hline \multicolumn{6}{|l|}{ Medications, N/\% } \\
\hline Anticoagulants & 792 & $3.1 \%$ & 27 & $6.0 \%$ & -13.9 \\
\hline Antiplatelets & 3443 & $13.3 \%$ & 94 & $20.9 \%$ & -20.3 \\
\hline Non-steroidal anti-inflammatory drugs & 1895 & $7.3 \%$ & 33 & $7.3 \%$ & 0.0 \\
\hline Selective serotonin reuptake inhibitors & 2772 & $10.7 \%$ & 61 & $13.6 \%$ & -8.9 \\
\hline Selective norepinephrine reuptake inhibitors & 324 & $1.3 \%$ & 7 & $1.6 \%$ & -2.5 \\
\hline \multicolumn{6}{|l|}{ Measures of healthcare utilization and overall health status } \\
\hline Distinct prescription medications taken, mean/SD & 3.2 & 3.0 & 3.9 & 3.5 & -21.5 \\
\hline Face-to-face primary care consultations, mean/SD & 9.4 & 8.0 & 10.7 & 9.7 & -14.6 \\
\hline Prior hospitalization, N/\% & 3201 & $12.3 \%$ & 59 & $13.1 \%$ & -2.4 \\
\hline
\end{tabular}

Notes: *Patient demographics were measured as of the index admission; comorbidities, medications, and measures of healthcare utilization and overall health status were measured over the 12-month period prior to the index admission; SD, standard deviation; StdDiff, standardized difference (a value $>|0.10|$ is indicative of imbalance between groups).

analyses including those for LOS and critical care utilization among patients undergoing lung surgery with baseline antiplatelet use ( $\mathrm{N}=441$ overall) and critical care utilization among patients undergoing mastectomy with baseline antiplatelet use ( $\mathrm{N}=1250$ overall).
With respect to complications and mortality, the odds, incidence proportions, and mean incremental difference in outcomes between patients with vs without in-hospital bleeding event were substantially higher in patients with baseline antiplatelet use as compared with those without 
Table 2 Characteristics of Index Admission and Surgery*

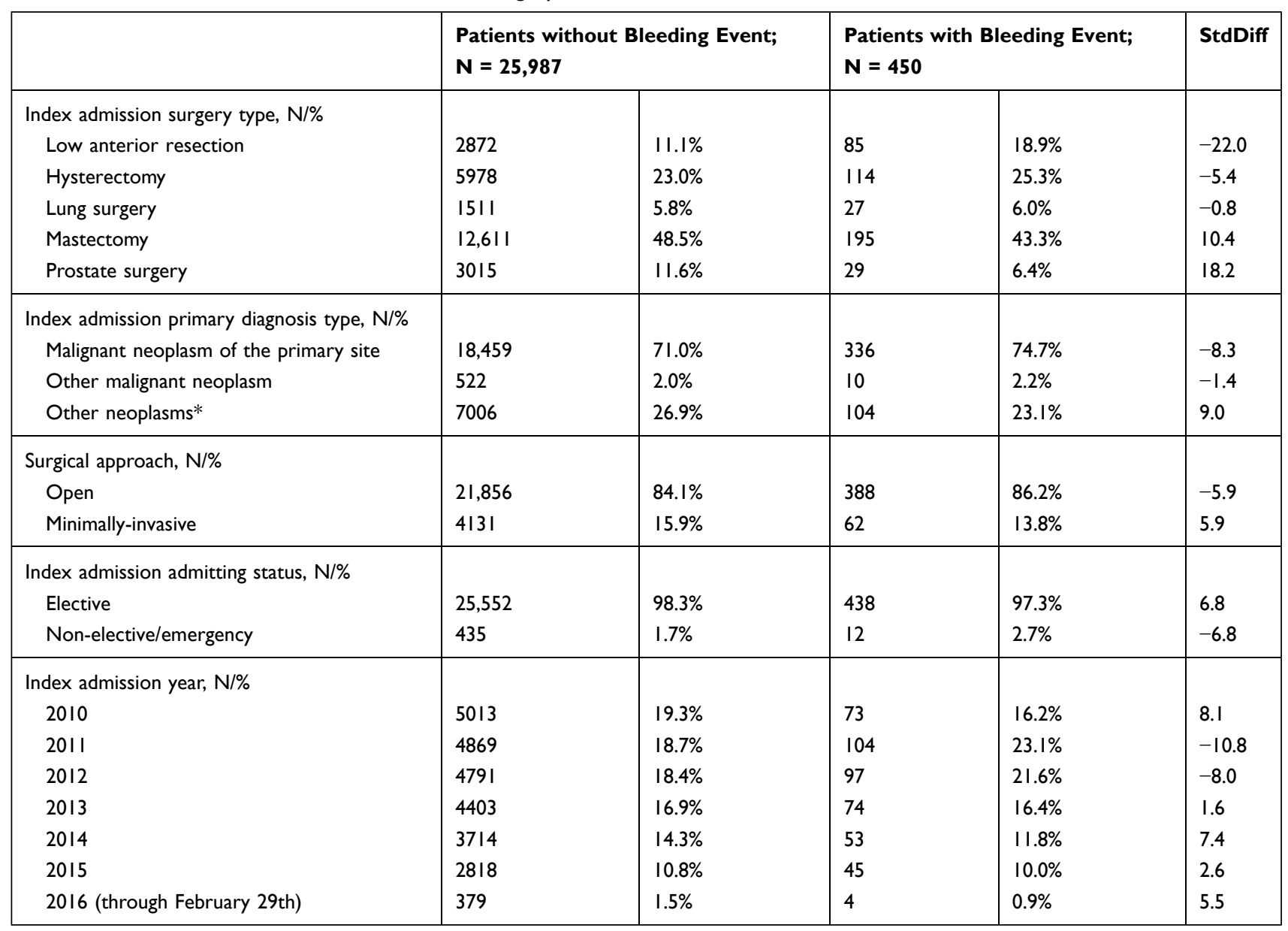

Notes: *Other neoplasms included benign neoplasms, carcinoma in situ, and neoplasms of uncertain behavior; StdDiff, standardized difference (a value $>\mid 0$. I0| is indicative of imbalance between groups).

baseline antiplatelet use. For example, whereas the odds ratio for mortality was $4.78(95 \% \mathrm{CI}=1.89-12.10)$ among patients without baseline antiplatelet use, it was $8.28(95 \%$ $\mathrm{CI}=2.86-23.99)$ among patients with baseline antiplatelet use.

\section{Discussion}

To our knowledge, this large, population-based study is the first to examine the association of in-hospital bleeding events with LOS, days spent in critical care, complications, and mortality among patients undergoing neoplasmdirected surgeries in English hospitals.

In the present study, the crude incidence proportion of in-hospital bleeding events for the index admission ranged from $1.0 \%(95 \% \mathrm{CI}=0.6-1.3 \%)$ among patients undergoing prostate surgery to $3.0 \%(95 \% \mathrm{CI}=2.3-3.6 \%)$ among patients undergoing low anterior resection; these estimates are generally comparable to those in the existing literature. The rate of bleeding complicating a procedure has been reported to be $2.5 \%$ for mastectomy, ${ }^{23} 0.5-2.6 \%$ for prostatectomy, ${ }^{24-26} 1.3-1.9 \%$ for thoracic surgery, ${ }^{27}$ $0.4 \%$ to $6.4 \%$ for hysterectomy, ${ }^{28-30}$ and $10.9 \%$ for low anterior resection $^{31}$ (for low anterior resection, the comparatively higher rate is likely explained by a broader definition of bleeding, which included not only hemorrhage and hematoma complicating a procedure but also seroma and acute post-hemorrhagic anemia). None of the aforementioned studies were based in UK, however, underscoring the unique contribution the present study adds to the literature.

We found that bleeding events were associated with substantial increases in both LOS and days spent in critical care, with MIDs in LOS ranging from 3.1 days $(95 \% \mathrm{CI}=1.1-6.3)$ among patients undergoing mastectomy to 5.7 days $(95 \% \mathrm{CI}$ $=3.6-8.2$ ) among patients undergoing low anterior resection, and MIDs in days spent in critical care ranging from 0.4 days $(95 \% \mathrm{CI}=0.03-0.27)$ among patients undergoing mastectomy to 6.5 days $(95 \% \mathrm{CI}=2.5-13.6)$ among patients 


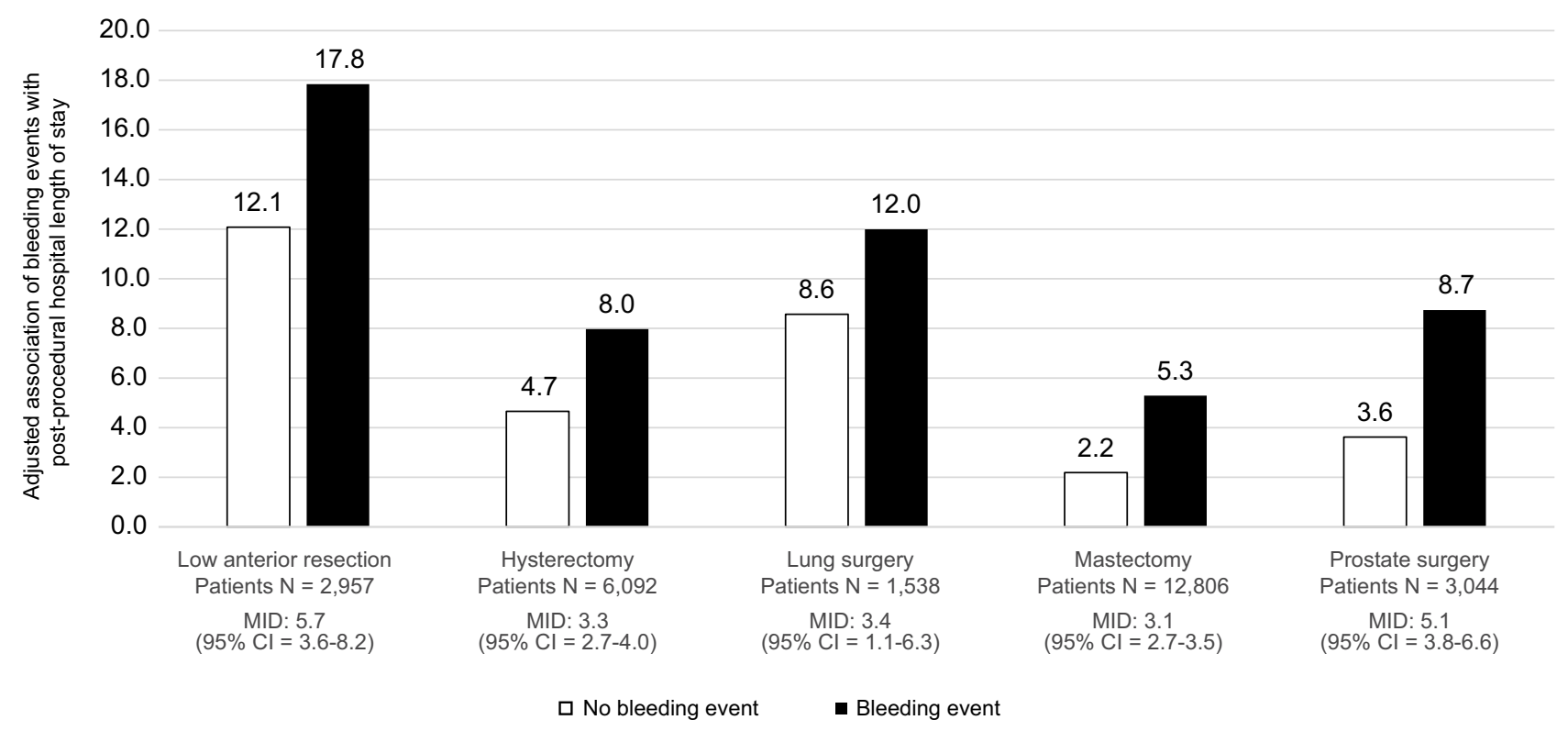

Figure 2 Adjusted association of bleeding events with post-procedural hospital length of stay*.

Note: *All difference in adjusted post-procedural hospital length of stay were statistically significant at $\mathrm{P}<0.05$.

Abbreviations: $\mathrm{Cl}$, confidence interval; $\mathrm{MID}$, mean incremental difference.

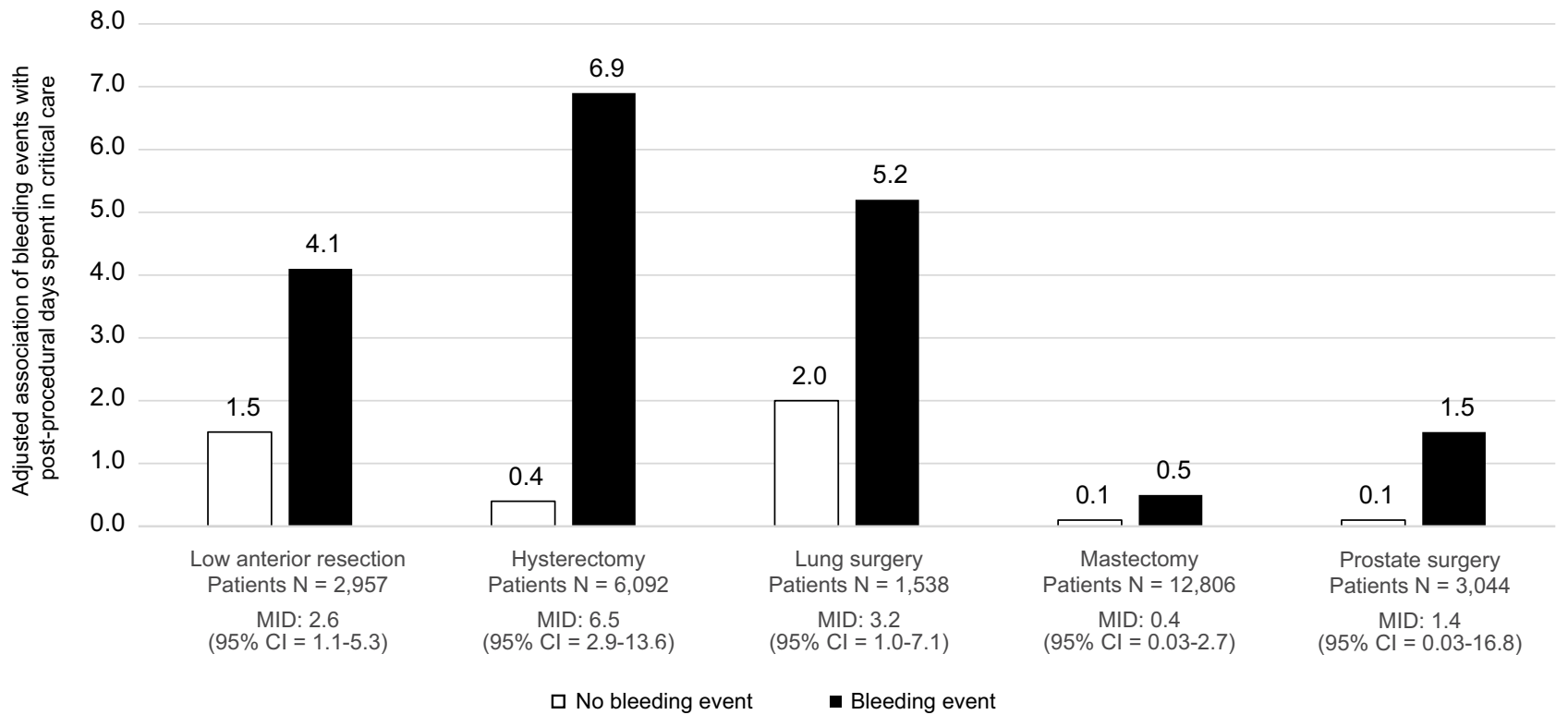

Figure 3 Adjusted association of bleeding events with post-procedural days spent in critical care*.

Note: *All difference in adjusted post-procedural days spent in critical care were statistically significant at $\mathrm{P}<0.05$.

Abbreviations: $\mathrm{Cl}$, confidence interval; MID, mean incremental difference.

undergoing hysterectomy. Although not directly comparable due to differences in country and surgical groupings, the present finding are consistent with those of a study conducted by Stokes and colleagues, ${ }^{2}$ which used hospital administrative data from the United States for patients undergoing various surgeries during 2006-2007. They reported that patients with bleeding-related complications or blood product transfusion experienced LOS that was 6 days longer (range 1.3 to 9.6 days depending on the surgery) and spent 2.8 more days in intensive care (range 0.1 to 7.4 days depending on the surgery) that were than those without such complications or transfusions.

In the present study, bleeding events were also associated with substantial increases in in-hospital infection, 


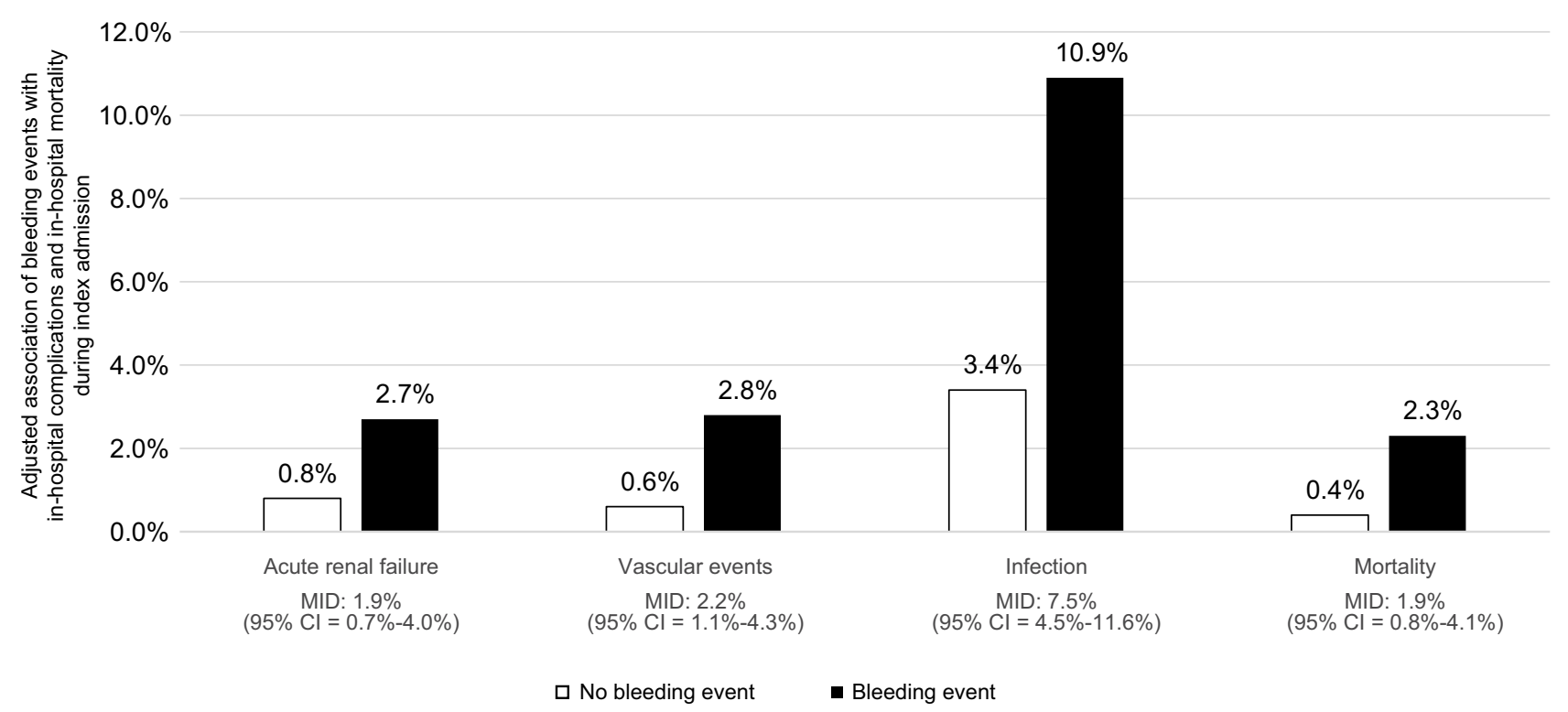

Figure 4 Adjusted association of bleeding events with in-hospital sequelae and in-hospital mortality during index admission*.

Notes: *Sequelae and mortality events were infrequent, which necessitated that we pool all patients from across the surgery groups when analyzing these outcomes; all difference in the incidence of sequelae and mortality were statistically significant at $P<0.05$.

Abbreviations: $\mathrm{Cl}$, confidence interval; $\mathrm{MID}$, mean incremental difference.

acute renal failure, vascular events, and mortality. These findings are consistent with several prior studies examining the association of transfusion with these outcomes, conducted primarily in the setting of cardiac surgery. ${ }^{7-11}$ The increased risk of these complications may serve as a mechanism by which bleeding events can lead to prolonged length of stay and more days spent in critical care.

In the post-hoc sensitivity analyses of the study outcomes in patients with vs without baseline use of antiplatelet medications, the association of in-hospital bleeding with the study outcomes was generally more pronounced among patients with baseline use of antiplatelet medications, particularly for complications and mortality; these finding may be indicative of more severe bleeding among such patients. Furthermore, these findings suggest that patients treated with antiplatelets who undergo these surgeries may be viewed as a high risk group for experiencing more severe outcomes related to in-hospital bleeding events.

\section{Limitations}

This study's results must be interpreted in context of its limitations, which are primarily driven by data that are unavailable in the HES-APC. We defined in-hospital bleeding events using administrative codes for haemorrhage and haematoma complicating a procedure or reoperation for surgical arrest of postoperative bleeding; however, as noted above, we were unable to identify blood product transfusions in this study because blood product transfusions are not well captured in the HESAPC database. ${ }^{17}$ Additionally, the HES-APC data do not contain information on the quantity of blood lost, which further limited our ability to characterize the severity of inhospital bleeding events. Although we cannot ultimately determine the severity of the bleeding events in the present study, their observed association with infections, vascular events, acute renal failure, and mortality (for each of which the use of blood product transfusion for the management of surgical bleeding has been implicated as a cause) suggest that many may have been severe enough to warrant blood product transfusion. Administrative databases are also subject to measurement error resulting from the use of diagnosis codes to define clinical variables. It is possible that some bleeding events may have occurred, but a corresponding diagnosis was not recorded, resulting in under-identification of bleeding events. In such a case, the results could both underestimate the true incidence of bleeding events and be biased towards the null hypothesis of no difference in outcomes between patients with vs those without bleeding events. Second, although we used multivariable analysis to account for a variety of patient and surgical characteristics, we cannot rule out the possibility of unmeasured confounders that affect both the risk of bleeding events and the outcomes; such unmeasured 
confounders could include the skill of the surgeon and his or her staff, concurrent implementation of risk mitigation strategies for bleeding events and prevention of complications, or clinical factors such as tumor size and surgical complexity. Third, the HES-APC does not have information on the use of products, such as hemostatic agents, that mitigate the impact of bleeding events. Future studies are needed to understand the extent to which hemostatic agents may attenuate the deleterious effects of bleeding events. We focused our analysis on the post-operative, inhospital setting; it is possible that in-hospital bleeding events may be associated with longer-term undesirable outcomes. Finally, the results are limited to English patients meeting the study selection criteria and may not necessarily be generalizable to other countries. Despite these limitations, the HES-APC and linkage with CPRDGOLD are an excellent source of large population-based data for epidemiologic research.

\section{Conclusions}

In conclusion, in-hospital bleeding events were associated with substantial increases in post-procedural LOS, days spent in critical care, and the risks of infection, vascular events, acute renal failure, and in-hospital mortality. This study quantifies a large clinical and economic burden associated with surgical bleeding among patients undergoing neoplasm-directed surgery within in English hospitals; improvements in prevention and mitigation strategies for surgical bleeding may yield substantial benefits.

\section{Data Sharing Statement}

The study data were collected from the Hospital Episode Statistics Admitted Patient Care (HES-APC) database and the Clinical Practice Research Datalink (CPRD) GOLD database. HES-APC and CPRD study data are publicly available under license from the UK Medicines and Healthcare Products Regulatory Agency (MHRA) after review by The Independent Scientific Advisory Committee for MHRA Database Research.

\section{Author Contributions}

All authors made substantial contributions to conception and design, acquisition of data, or analysis and interpretation of data; took part in drafting the article or revising it critically for important intellectual content; agreed to submit to the current journal; gave final approval of the version to be published; and agree to be accountable for all aspects of the work.

\section{Funding}

This study was funded by Johnson \& Johnson.

\section{Disclosure}

Stephen S. Johnston, Nadine Jamous, Sameer Mistry, Gaurav Gangoli, Walter Danker, and Eric Ammann are currently employed or were employed by Johnson \& Johnson at the time this study was conducted. Gaurav Gangoli and Walter Danker were affiliated with Ethicon at the time the study was conducted. Eric Ammann was affiliated with Janssen at the time the study was conducted. Simran Jain was employed by $\mathrm{Mu}$ Sigma at the time the study was conducted. Simran Jain and Kingsley Hampton were consultants to Johnson \& Johnson at the time that this research was conducted. The authors report no other conflicts of interest in this work.

\section{References}

1. Shander A. Financial and clinical outcomes associated with surgical bleeding complications. Surgery. 2007;142(4 Suppl):S20-5. doi:10.1016/j.surg.2007.06.025

2. Stokes ME, Ye X, Shah M, et al. Impact of bleeding-related complications and/or blood product transfusions on hospital costs in inpatient surgical patients. BMC Health Serv Res. 2011;11(1):135. doi:10.1186/1472-6963-11-135

3. Corral M, Ferko N, Hollmann S, Broder MS, Chang E. Health and economic outcomes associated with uncontrolled surgical bleeding: a retrospective analysis of the premier perspectives database. Clinicoecon Outcomes Res. 2015;7:409-421. doi:10.2147/CEOR. S86369

4. Cata JP, Vijaya Gottumukkala V. Blood loss and massive transfusion in patients undergoing major oncological surgery: what do we know? ISRN Anesthesiol. 2012;2012:1-11. doi:10.5402/2012/918938

5. Johnstone C, Rich SE. Bleeding in cancer patients and its treatment: a review. Ann Palliat Med. 2018;7(2):265-273. doi:10.21037/ apm.2017.11.01.

6. Pereira J, Phan T. Management of bleeding in patients with advanced cancer. Oncologist. 2004;9(5):561-570. doi:10.1634/ theoncologist.9-5-561

7. Murphy GJ, Reeves BC, Rogers CA, Rizvi SI, Culliford L, Angelini GD. Increased mortality, postoperative morbidity, and cost after red blood cell transfusion in patients having cardiac surgery. Circulation. 2007;116(22):2544-2552. doi:10.1161/ CIRCULATIONAHA.107.698977

8. Reeves BC, Murphy GJ. Increased mortality, morbidity, and cost associated with red blood cell transfusion after cardiac surgery. Curr Opin Anaesthesiol. 2008;21(5):669-673. doi:10.1097/ ACO.0b013e32830dd087.

9. Dellinger EP, Anaya DA. Infectious and immunologic consequences of blood transfusion. Crit Care. 2004;8(Suppl 2):S18-S23. doi: $10.1186 / \mathrm{cc} 2847$

10. Karkouti K. Transfusion and risk of acute kidney injury in cardiac surgery. Br J Anaesth. 2012;109(Suppl 1):i29-38. doi:10.1093/bja/ aes 422

11. Glance LG, Dick AW, Mukamel DB. Association between intraoperative blood transfusion and mortality and morbidity in patients undergoing noncardiac surgery. Anesthesiology. 2011;114 (2):283-292. doi:10.1097/ALN.0b013e3182054d06. 
12. Medicines and Healthcare Products Regulatory Agency. CPRD linked data. Available from: https://cprd.com/linked-data\#HES\% 20Admitted\%20Patient\%20Care\%20data. Accessed July 11, 2019.

13. Padmanabhan S, Carty L, Cameron E, Ghosh RE, Williams R, Strongman H. Approach to record linkage of primary care data from clinical practice research datalink to other health-related patient data: overview and implications. Eur J Epidemiol. 2019;34(1):91-99. doi:10.1007/s10654-018-0442-4.

14. Medicines and Healthcare Products Regulatory Agency. Hospital episode statistics (HES) admitted patient care (APC) data dictionary (set 17). Available from: https://www.cprd.com/sites/default/files/ HES\%20APC\%20Data\%20Dictionary\%20set\%2017_1.pdf. Accessed July 11, 2019.

15. Medicines and Healthcare Products Regulatory Agency. CPRD GOLD data specification. Available from: https:/www.cprd.com/ sites/default/files/CPRD\%20GOLD\%20Fu11\%20Data\% 20Specification\%20v2.0_0.pdf. Accessed July 11, 2019.

16. Medicines and Healthcare Products Regulatory Agency. Bibliography. Available from: https:/cprd.com/bibliography. Accessed July 11, 2019.

17. Llewelyn CA, Wells AW, Amin M, et al. The EASTR study: a new approach to determine the reasons for transfusion in epidemiological studies. Transfus Med. 2009;19(2):89-98. doi:10.1111/j.13653148.2009.00911.x

18. Quan H, Sundararajan V, Halfon P, et al. Coding algorithms for defining comorbidities in ICD-9-CM and ICD-10 administrative data. Med Care. 2005;43(11):1130-1139. doi:10.1097/01. mlr.0000182534.19832.83

19. Flury BK, Riedwyl H. Standard distance in univariate and multivariate analysis. Am Stat. 1986;40:249-251.

20. Manning WG, Mullahy J. Estimating log models: to transform or not to transform? J Health Econ. 2001;20(4):461-494. doi:10.1016/ S0167-6296(01)00086-8

21. Park RE. Estimation with heteroscedastic error terms. Econometrica. 1996;34(4):888. doi:10.2307/1910108
22. Muller CJ, MacLehose RF. Estimating predicted probabilities from logistic regression: different methods correspond to different target populations. Int J Epidemiol. 2014;43(3):962-970. doi:10.1093/ije/ dyu029

23. Nwaogu IY, Bommarito K, Olsen MA, Margenthaler JA. Economic impact of bleeding complications after mastectomy. J Surg Res. 2015;199(1):77-83. doi:10.1016/j.jss.2015.03.084

24. Koc E, Canda AE. Robotic urologic surgery complications. Minim Invasive Surg. 2018;2(4):7. doi:10.20517/2574-1225.2017.33

25. Alibhai SM, Leach M, Tomlinson G, et al. 30-day mortality and major complications after radical prostatectomy: influence of age and comorbidity. J Natl Cancer Inst. 2005;97(20):1525-1532. doi:10.1093/jnci/dji313

26. Williams SB, Prasad SM, Weinberg AC, et al. Trends in the care of radical prostatectomy in the United States from 2003 to 2006. BJU Int. 2011;108(1):49-55. doi:10.1111/j.1464-410X.2010.09822.x

27. Kent M, Wang T, Whyte R, Curran T, Flores R, Gangadharan S. Open, video-assisted thoracic surgery, and robotic lobectomy: review of a national database. Ann Thorac Surg. 2014;97(1):236-242. doi:10.1016/j.athoracsur.2013.07.117.

28. Holub Z, Jabor A. Laparoscopic management of bleeding after laparoscopic or vaginal hysterectomy. JSLS. 2004;8(3):235-238.

29. Topsoee MF, Ibfelt EH, Settnes A. The Danish hysterectomy and hysteroscopy database. Clin Epidemiol. 2016;8:515-520. doi:10.2147/CLEP.S99465

30. Osler M, Daugbjerg S, Frederiksen BL, Ottesen B. Body mass and risk of complications after hysterectomy on benign indications. Hum Reprod. 2011;26(6):1512-1518. doi:10.1093/humrep/der060

31. Ammann EM, Goldstein LJ, Nagle D, Wei D, Johnston SS. A dual-perspective analysis of the hospital and payer-borne burdens of selected in-hospital surgical complications in low anterior resection for colorectal cancer. Hosp Pract. 2019;47(2):80-87. doi:10.1080/ 21548331.2019.1568718
ClinicoEconomics and Outcomes Research

\section{Publish your work in this journal}

ClinicoEconomics and Outcomes Research is an international, peerreviewed open-access journal focusing on Health Technology Assessment, Pharmacoeconomics and Outcomes Research in the areas of diagnosis, medical devices, and clinical, surgical and pharmacological intervention. The economic impact of health policy and health systems

\section{Dovepress}

organization also constitute important areas of coverage. The manuscript management system is completely online and includes a very quick and fair peer-review system, which is all easy to use. Visit $\mathrm{http}: / / \mathrm{www}$.dovepress.com/testimonials.php to read real quotes from published authors.

Submit your manuscript here: https://www.dovepress.com/clinicoeconomics-and-outcomes-research-journal 